\title{
Soft X-ray Synchrotron Radiation Investigations of Actinide Materials Systems Utilizing X-ray Emission Spectroscopy and Resonant Inelastic X-ray Scattering
}

\author{
D. K. Shuh, ${ }^{1}$ S. M. Butorin, ${ }^{2}$ J.-H. Guo, ${ }^{3}$ and J. Nordgren ${ }^{2}$ \\ ${ }^{1}$ Chemical Sciences Division, Lawrence Berkeley National Laboratory (LBNL), Berkeley, \\ California 94720 USA \\ ${ }^{2}$ Department of Physics, Uppsala University, Box 530, S-751 21 Uppsala, Sweden \\ ${ }^{3}$ Advanced Light Source Division, Lawrence Berkeley National Laboratory (LBNL), Berkeley, \\ California 94720 USA
}

\begin{abstract}
Synchrotron radiation (SR) methods have been utilized with increasing frequency over the past several years to study topics in actinide science, ranging from those of a fundamental nature to those that address a specifically-targeted technical need. In particular, the emergence of microspectroscopic and fluorescence-based techniques have permitted investigations of actinide materials at sources of soft x-ray SR. Spectroscopic techniques with fluorescence-based detection are useful for actinide investigations since they are sensitive to small amounts of material and the information sampling depth may be varied. These characteristics also serve to simplify both sample preparation and safety considerations. Examples of investigations using these fluorescence techniques will be described along with their results, as well as the prospects for future investigations utilizing these methodologies.
\end{abstract}

\section{INTRODUCTION}

The state-of-the-art understanding of the electronic structure, surface chemistry, and interfacial properties of actinide materials lags substantially behind that of other materials systems in part because of radiological safety issues that have prevented the actinide science community from taking advantage of SR methodologies in the soft x-ray region, which revolutionized the approach to surface chemistry/physics twenty years ago. A major unresolved question in the properties of actinide materials is the nature of the $5 f$ electrons and their exact role in chemical bonding which determines their materials chemistry. The radiological safety concerns arise due to the difficulty of safely handling, preparing, and performing measurements on actinide materials under vacuum shared with beamlines and soft $\mathrm{x}$-ray SR storage rings.

One of the most powerful techniques for studying electronic structure is photoelectron spectroscopy employing tunable soft x-ray SR. Determining the features attributable solely to $5 f$ electrons is difficult if a photoelectron spectrum is collected at a single photon energy. The ability to perform resonant photoemission measurements at the $5 d$ threshold, where $5 f$ emission is selectively enhanced, is crucial to better understand actinide materials. Tunable light near the actinide $5 d$ absorption edges ( $~ 98 \mathrm{eV}$ for $\mathrm{U})$ makes the identification possible due to the large Fano resonances. Recently, significant results from resonant photoemission investigations of metallic Pu have been obtained at the Advanced Light Source (ALS), as well as a tunable light source.[1-3] However, soft x-ray SR photoemission studies are complicated by the need to handle radioactive materials at the SR facility to prepare contaminant-free actinide surfaces.

The soft X-ray SR fluorescence techniques, X-ray emission spectroscopy (XES) and resonant inelastic $x$-ray scattering (RIXS), combined with microbeam capabilities and flux available from a third generation soft x-ray SR source, provide a unique opportunity to extend the understanding of chemical bonding in f-electron systems.[4-6] The information from these fluorescence techniques are complementary to photoemission and do not suffer from core hole effects. Of special importance is RIXS, which can provide specific electronic structure information to characterize chemical bonding. The fluorescence techniques allow safe handling and practical experiments with extremely small amounts of non-pristine, bulk actinide materials since they circumvent actinide materials quality issues that can complicate electron-detecting 
photoemission experiments. Sample materials are also not limited to conducting materials. Furthermore, using grazing incidence or having solely a surface species, the fluorescence techniques become surface sensitive.

The $\mathrm{x}$-ray emission process is illustrated in Figure 1 and is characterized by radiative deexcitation of electrons into the core hole states. These transitions are governed by strong dipole selection rules and since the valence band states have a significant energy width, the resulting XES spectrum corresponds to an element specific valence band partial density of states resolved by orbital angular momentum components. In the special case when the excitation energy is close to the elemental absorption threshold, the electron occupies the conduction band and it is intuitive to consider this as a RIXS process. The RIXS energy loss features can be understood as band to band transitions, both dipole allowed (charge-transfer) and dipole forbidden.[4-5]

RIXS measurements at the actinide $5 d$ thresholds provides an opportunity to study elementary excitations, the electronic structures, and the materials chemistries of actinide compounds due to the higher resolution of such experiments compared with those at the actinide $3 \mathrm{~d}$ and $4 \mathrm{~d}$ thresholds.[4,7] In addition, RIXS at the $5 d$ threshold is sensitive to the valence and chemical speciation of $\mathrm{U}$, contrasting to $\mathrm{x}$-ray absorption spectroscopy (XAS). The $5 d$ core-hole lifetime broadening is large, limiting the utility of XAS spectra since the substantial smearing of the spectral structures hampers the analysis of the chemical state and the chemical environment of $U$ in various compounds.[8] The superior resolution of RIXS and the enhancement of transitions to low-lying excited states are especially useful. RIXS spectroscopy provides signatures of distinct transitions representing electronic excitations within the $5 f$ shell and can have characteristic profiles for specific oxidation states (e.g., U(IV) versus U(VI)).[4]

\section{EXPERIMENTAL}

XES experiments were performed at the spherical grating monochromator undulator Beamlines (BLs) 7.0 and 8.0 of the ALS at LBNL. The XES and RIXS spectra were collected utilizing the resident grazing-incidence grating XES spectrometers with two-dimensional detectors.[9-10] The incidence angle of the photon beam was approximately $15^{\circ}$ and $45^{\circ}$ from the surface normal at BLs-7.0 and 8.0, respectively. Both XES spectrometers were arranged at $90^{\circ}$ with respect to the incident beam. In general, the bandwidth of excitations was about $65 \mathrm{meV}$ when operating around $100 \mathrm{eV}$. A representative total energy resolution for the RIXS data acquired on BL-7 was estimated from the FWHM of the elastic scattering peak and was equal to approximately $160 \mathrm{meV}$. The experimental procedures followed the safety protocols developed in conjunction with the LBNL EH\&S Radiation Protection Group and the ALS.[11]
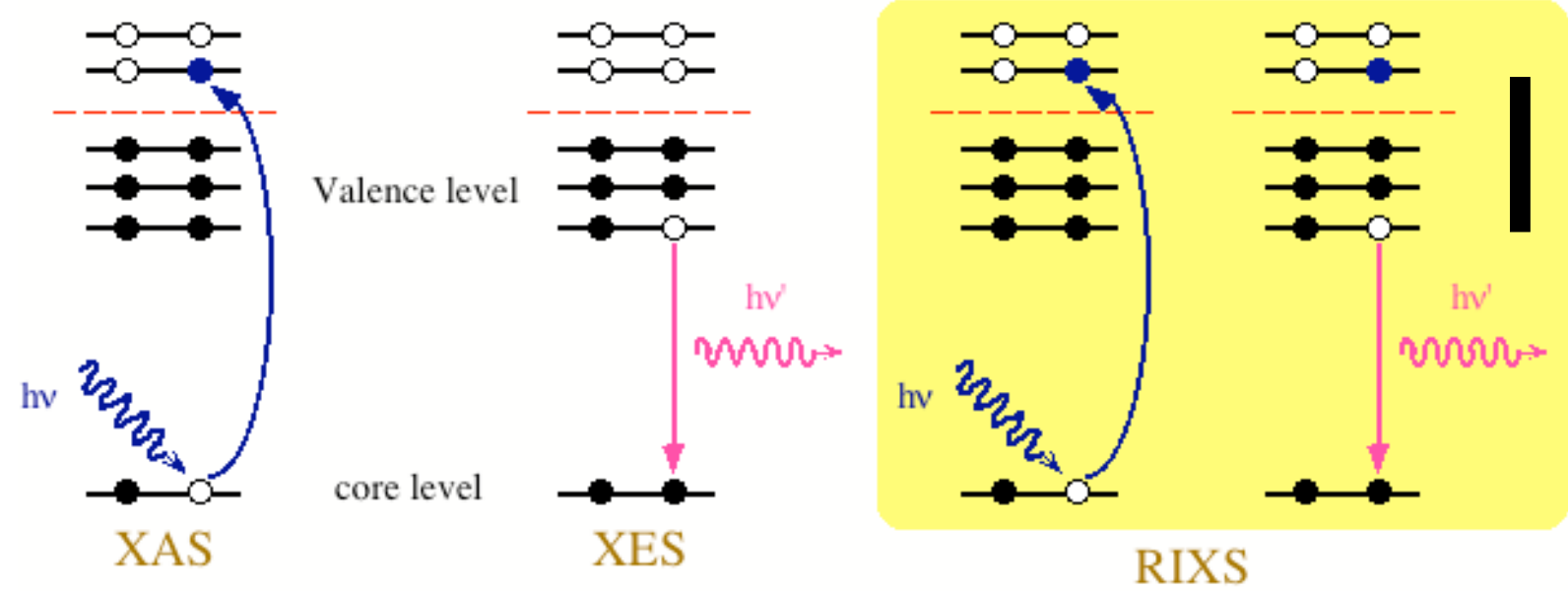

Figure 1. Schematic of the x-ray absorption spectroscopy (XAS), x-ray emission spectroscopy (XES), and resonant inelastic x-ray scattering (RIXS) processes. The energy difference 
represented by the bar on the right of the shaded panel and equal to $h v-h v '$, is observed as a loss feature from the energy of the elastic scattering feature (hv) in the RIXS spectrum.

\section{RESULTS AND DISCUSSION}

A prototypical set of RIXS measurements at an actinide $5 d$ threshold have been performed on solid $\mathrm{UF}_{4}$ (Noah Chemical) at BL-7.0 of the ALS and the spectra are shown in Figure 2. The RIXS spectra were obtained for different incident photon energies in the U pre-5dthreshold region as indicated on the XAS spectrum of Figure 2 and probe the $f-f$ excitations in the actinide system. The experimental x-ray inelastic scattering loss features (upper spectra) and the broadened, calculated emission (lower spectra) are shown on the energy loss scale relative to the elastic feature for comparison. The assignment of the sharp inelastic loss features to $f-f$ transitions is supported by the results of atomic multiplet calculations using only the resonant term from the modified Kramers-Heisenberg equation that treats RIXS as a second order optical process.[4] In the calculations, the varying lifetime of core-excited states due to autoionization via the $5 d-5 f 5 f$ super Coster-Kronig decay was taken into account and only autoionization into the continuum of $g$ symmetry considered since it is the dominant path. Matrix elements were obtained from Cowan's program and the complete details of the calculations can be found in Ref. 4.

The calculations reproduce all of the spectral structures well, especially the enhancement of loss peak at about $-1.2 \mathrm{eV}$ with increasing photon energy that originates for transitions into the ${ }^{1} G_{4}$ state. Changes in the intensity of the $f-f$ transitions are observed from spectrum a to b in accordance with the calculations. However, in spectra $\mathrm{c}$ and $\mathrm{d}$, the calculated intensities are about three times larger than observed. This discrepancy between experiment and the theoretical calculation may originate from the normalization procedure for the experimental spectra accounting for variations in the incident photon flux. The intensity of the elastic peak was used as a reference in this procedure and may contain some contribution of diffuse scattering that varies with excitation energies.

RIXS measurements of the suite of common U oxides has provided detailed information about the characteristics of RIXS for actinide materials. Incident excitation energies close to the $\mathrm{U} 5 d$ threshold enhance the cross-sections for inelastic scattering and probe the states of $5 f$ symmetry. To selectively examine the intra-ionic $f-f$ transitions, excitations at about $100 \mathrm{eV}$ yield the most spectral weight. At higher incident photon beam energies near the main U $5 d$ absorption edge (e.g., $115 \mathrm{eV}$ ) inter-ionic excitations of charge-transfer character, such as ligand $2 p-\mathrm{U} 5 f$ charge-transfer, dominate the RIXS spectra. The spectral pattern of intra-ionic $f-f$ excitations is mainly determined by the formal valence of $U$, and in turn, the charge-transfer transitions depend strongly on the chemical environment of U. Thus, these two characteristics make RIXS a valuable tool to investigate the electronic structure and materials chemistry of actinides.

\section{Studies of Uranium(VI) Reduction on Iron Surfaces}

The RIXS technique has been used at BL-7.0 of the ALS to investigate the chemical reaction of dilute $\mathrm{U}(\mathrm{VI})$ solutions at iron surfaces. The objective was to evaluate the interaction that would occur if $\mathrm{UO}_{2}$ were to oxidize to $\mathrm{U}(\mathrm{VI})$ inside of the cast steel canister used in the Swedish nuclear fuel repository.[12] In this situation, any reduction of U(VI) on the Fe surface should result in an appearance of characteristic RIXS structures. Of course, these structures are absent in the spectra of $\mathrm{UO}_{3}$ or any other $\mathrm{U}(\mathrm{VI})$ species due to the formal $5 f^{\dagger}$ configuration of the $\mathrm{U}(\mathrm{VI})$ ion. RIXS measurements at $100 \mathrm{eV}$ of bulk polished Fe coupons exposed to $2 \mathrm{ppm} \mathrm{U}(\mathrm{VI})$ had several locations (but not all) that yielded distinct structures with energy losses of -0.8 and $-1.2 \mathrm{eV}$ from the elastic peak. These loss features were compared directly to those of a $\mathrm{UO}_{2}$ reference and represent $f-f$ transitions from a U(IV) core, indicating reduction of U(VI) at the surface. However, RIXS spectra obtained in the region of charge-transfer transitions (115 eV) characteristic of the $U$ chemical environment differ, suggesting that the U(IV) species on the Fe coupon is not necessarily $\mathrm{UO}_{2}$. Figure 3 shows the successfully tested vacuum-compatible, 
transferable liquid reaction cell that had a thin Fe film grown on the backside of the silicon nitride isolation window followed by in-situ exposure to a U(VI) solution. 

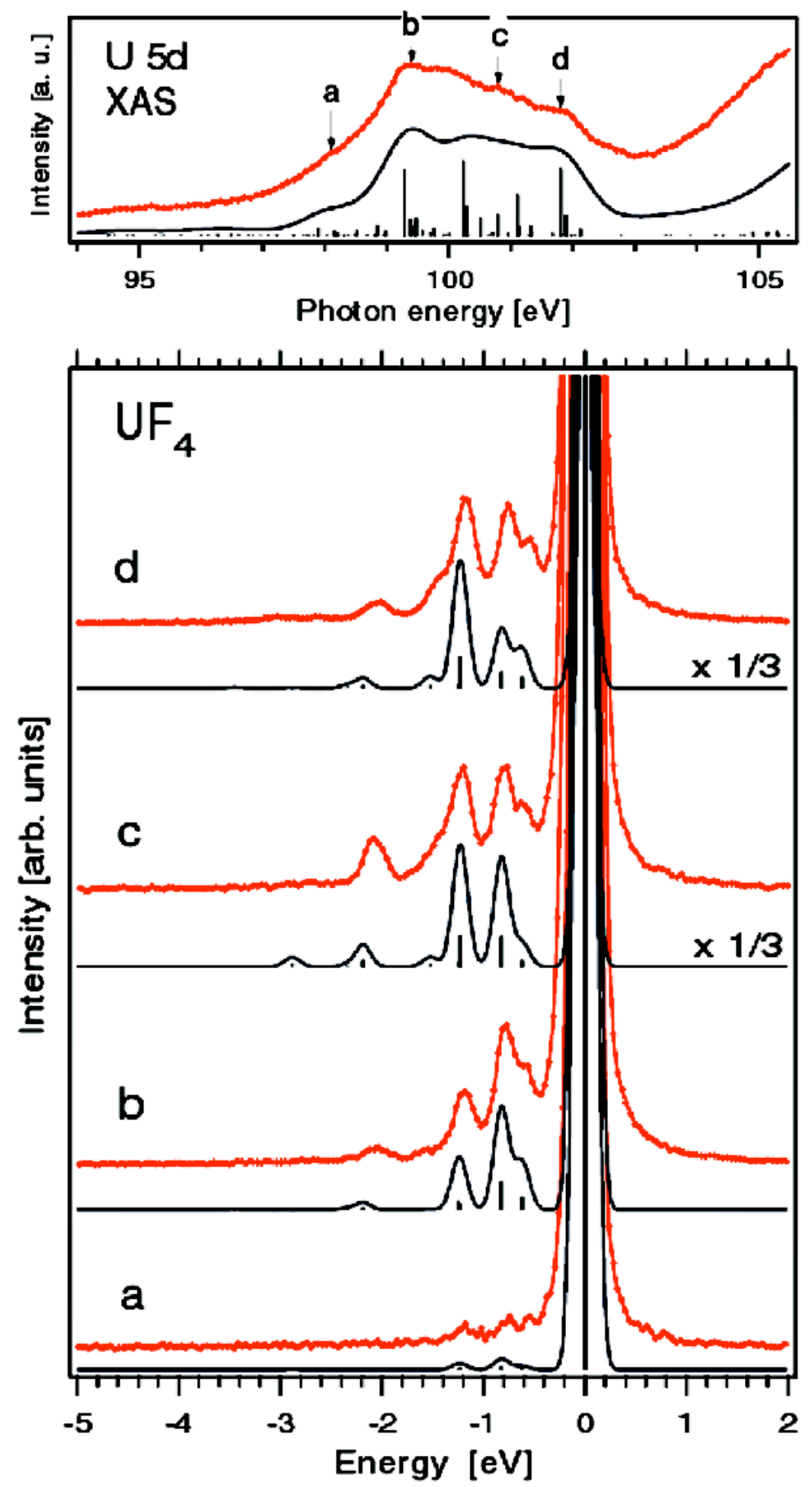

Figure 2. The lower panel shows experimental resonant x-ray scattering spectra (dotted upper solid traces) from $\mathrm{UF}_{4}$ collected as a function of excitation energies near the $\mathrm{U} 5 \mathrm{~d}$ threshold along with the results of broadened atomic multiplet calculations (lower solid lines) for the U(IV) ion. The excitation energies used are indicated by arrows on the total electron yield spectrum at the $\mathrm{U} 5 \mathrm{~d}$ absorption edge shown in the upper panel (uppermost trace experiment; lowermost trace calculation). 

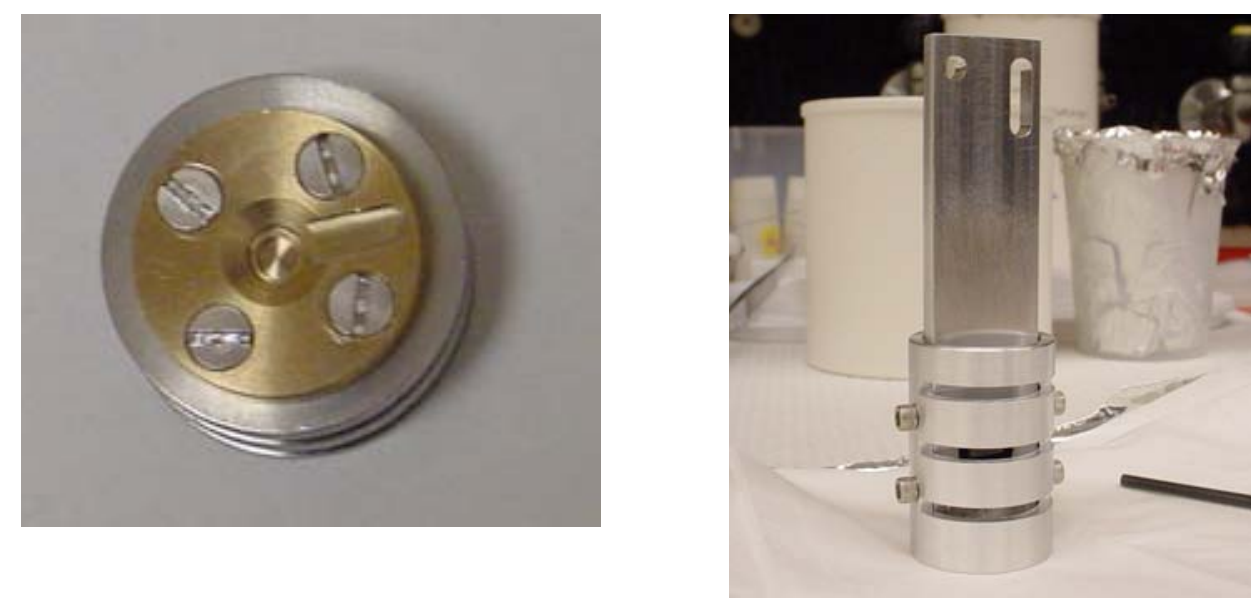

Figure 3. Sample holders used to perform the RIXS measurements of in-situ uranium(VI) adsorption on thin iron films, Np sorption on iron coupons, and amorphous $\mathrm{NpO}_{2}$ at BL-7.0 of the ALS. The liquid cell shown on the left (without the silicon nitride window) was utilized for in-situ U studies, whereas the assembly on the right was used for the Np investigations. The diameters of the transferable puck and the slotted can are both $25.4 \mathrm{~mm}$.

\section{Studies of the Borides of Uranium and Thorium}

The use of the fluorescence emission spectroscopies in the soft X-ray region also provides the ability to utilize the $\mathrm{K}$ edges of the light element constituents of actinide materials in concert with the actinide $5 d$ edge. For actinide compounds possessing only light element constituents, the complete element specific contributions to electronic structure can be elucidated. Both XES and RIXS methodologies have been used to investigate the unique electronic structures of $U$ and Th borides at BL-8.0 of the ALS.[13] In addition to the regular and common borides of these actinide elements, several novel borides have also been examined. Although the results are preliminary and are in the process of being interpreted, in some instances there are similarities between the spectra of the actinide borides and those observed for $\mathrm{MgB}_{2}$.[14]

\section{Studies of Np Materials}

The first measurements of a transuranic material, $\mathrm{NpO}_{2}$, by the fluorescence emission techniques has been recently performed at the ALS.[15] This investigation utilized microgram amounts of $\mathrm{Np}$, the LBNL safety procedures, and a specialized sample holder for the radioactive materials. The sample holder is shown in Figure 3. The holder is designed to prevent material from contaminating the vacuum chamber should it unexpectedly come loose, while permitting the transmission of excitation and signal beams. The RIXS spectra obtained from the $\mathrm{NpO}_{2}$ reference material, once again collected at several excitation energies, have a number of sharp inelastic loss features. Preliminary comparison to calculated spectra yields good agreement, although the match is not as good as for the U spectra shown in Figure 2.

\section{CONCLUSIONS}

The techniques of soft x-ray SR XES and RIXS can provide valuable fundamental information about the electronic structure, elementary excitations, and the chemistries of actinide materials. These methods are excellent element-specific tools for determining oxidation state and the local chemical environments of actinides in their most straightforward applications. Furthermore, the results can be successfully interpreted using an appropriate theoretical framework that is also useful from the actinide science perspective. Although much of the initial promise and importance of these techniques for actinide materials have been based on results 
from $\mathrm{U}$ materials, recent studies of $\mathrm{Np}$ establish that these methods will be of great utility for all actinides. The sensitivity of the photon-in, photon-out techniques permits experimentation with very small amounts of material and since the methods can be bulk sensitive, these characteristics simplify both sample preparation and safety. Furthermore, the techniques are versatile since they can be surface sensitive and are currently compatible to a limited degree with the use of novel sample cells for actinides. Future soft X-ray SR fluorescence emission spectroscopy investigations are anticipated to study a range of topics pertinent to plutonium materials, transplutonium materials, actinide interfacial species, and surface science of actinide materials.

\section{ACKNOWLEDGEMENTS}

This work was supported by the Director, Office of Science, Office of Basic Energy Sciences, Division of Chemical Sciences, Geosciences, and Biosciences of the U.S. Department of Energy (DOE) at LBNL under Contract No. DE-AC03-76SF00098. This work was also supported by the European Union via contract FIKW-CT-2000-00019, by the Swedish Research Council, and Göran Gustafsson Foundation for Research in Natural Sciences and Medicine. The ALS is operated by the DOE, Director, Office of Basic Energy Sciences (BES), Division of Material Sciences under Contract No. DE-AC03-76SF00098 at LBNL.

\section{REFERENCES}

1. J. Terry, R. K. Schulze, J. Lashley, J. D. Farr, T. Zocco, K. Heinzelman, E. Rotenberg, D. K. Shuh, G. Van der Laan, and J. G. Tobin, Surf. Sci. 499, 141 (2002).

2. J. G. Tobin, B. W. Chung, G. D. Wadddill, R. K. Schulze, J. Terry, J. D. Farr, T. Zocco, D. K. Shuh, K. Heinzelman, E. Rotenberg, G. Van der Laan, Phys. Rev. B 155109 (2003).

3. A. J. Arko, J. J. Joyce, L. Morales, J. Wills, J. Lashley, F. Wastin, and J. Rebizant, Phys. Rev. B 62, 1773 (2000).

4. S. M. Butorin, J. Electr. Spectros. Rltd. Phenomena 110-111, 213 (2000).

5. K. E. Smith, C. McGuiness, J. Downes, L. Duda, P. Ryna, C. Stagarescu, J. Guo, J. Nordgren, and S. D. Hulbert, Synchrotron Rad. News 15, 11 (2002).

6. Soft X-ray Science in the Next Millenium: The Future of Photon-In/Photon-Out Experiments: An Assessment of the Scientific and Technological Opportunities and the Instrumentation and Synchrotron Radiation Facility Needs at the Beginning of the New Millenium, Fall Creek Falls State Park, Pikeville, TN, (March, 2000).

7. S. M. Butorin, D. C. Mancini, J. H.-Guo, N. Wassdahl, J. Nordgren, M. Nakazawa, S. Tanaka, T. Uozumi, A. Kotani, Y. Ma, K. Miyano, S. H. Southworth, B. A. Karlin, and D. K. Shuh, Phys. Rev. Lett. 77, 574 (1996).

8. G. Kalkowski, G. Kaindl, W. D. Brewer, and W. Krone, Phys. Rev. B 35, 2667 (1987).

9. T. Warwick, P. Heimann, D. Mossessian, W. McKinney, and H. Padmore, Rev. Sci. Instrum. 66, 2037 (1995); J. Nordgren, G. Bray, S. Cramm, R. Nyholm, J.-E. Rubensson, and N. Wassdahl, Rev. Sci. Instrum. 60, 1690 (1989).

10. J. J. Jia, T. A. Callcott, J. Yurkas, A. W. Ellis, F. J. Himpsel, M. G. Samant, J. Stöhr, D. Ederer, J. A. Carlisle, E. A. Hudson, L. J. Terminello, D. K. Shuh, and R. C. C. Perera, Rev. Sci. Instrum. 66, 1394 (1995).

11. D. K. Shuh, N. M. Edelstein, and J. J. Bucher, Safety Procedures for Electron Spectroscopy of Actinides at the ALS, LBNL-39909, (1997).

12. S. M. Butorin, D. K. Shuh, K. Kvashnina, I. Soroka, K. Ollila, J.-H. Guo, K. E. Roberts, L. Werme, and J. Nordgren, Mater. Res. Soc. Symp. Proc., Kalmar, Sweden, in press (2003).

13 A. Lupinetti, K. Abney, D. K. Shuh, S. M. Butorin, J.-H. Guo, J. D. Denlinger, M. K. Gilles, and T. Tyliszczak, (to be published).

14. C. McGuiness, K. E. Smith, S. M. Butorin, J. H. Guo, J. Nordgren, T. Vogt, G. Schneider, J. Reilly, J. J. Tu, P. D. Johnson, and D. K. Shuh, Europhysics Lett. 56, 112 (2001).

15. S. M. Butorin, Y. Albinsson, D. K. Shuh, J.-H. Guo, L. Werme, and J. Nordgren, (to be published). 